\title{
GAME - A small mission concept for high-precision astrometric test of General Relativity
}

\author{
A. Vecchiato ${ }^{1}$, Mario Gai ${ }^{1}$, Paolo Donati ${ }^{2,1}$, Roberto Morbidelli ${ }^{1}$, \\ Mario G. Lattanzi ${ }^{1}$ and Mariateresa Crosta ${ }^{1}$ \\ ${ }^{1}$ Istituto Nazionale di Astrofisica - Osservatorio Astronomico di Torino, \\ Via Osservatorio 20, I-10025 Pino Torinese (TO), Italy \\ email: vecchiato@oato.inaf.it \\ ${ }^{2}$ Università di Torino - Dipartimento di Fisica, \\ via Giuria 1, I-10125 Torino, Italy
}

\begin{abstract}
GAME (Gamma Astrometric Measurement Experiment) is a concept for a small mission whose main goal is to measure from space the $\gamma$ parameter of the Parameterized PostNewtonian formalism, Will (2001)) A satellite, looking as close as possible to the Solar limb, measures the gravitational bending of light in a way similar to that followed by past experiments from the ground during solar eclipses. In the cited formalism, deviations of the $\gamma$ parameter from unity are interpreted as deviations from the predictions of General Relativity which are foreseen by several competing theories of gravity. In the present theoretical scenario, such deviations are expected to appear in the range between $10^{-5}$ and $10^{-7}$. The most stringent experimental constraints available up to now are those of the Cassini mission, that gives $1-\gamma \lesssim 10^{-} 5$ Bertotti et al. (2003), while future space missions are expected to reach the $10^{-7}$ level of accuracy. (Vecchiato et al. (2003), Turyshev et al. (2004), Ni (2008))

Preliminary simulations have shown that the expected final accuracy of GAME can reach the $10^{-7}$ level, or better if the mission profile can be extended to fit a larger budget Vecchiato et al. (2009), Gai et al. (2009). This work, which has recently been extended to better assess the mission performances, has confirmed the previous results and has given indications on how further improve various aspects of the mission profile.

Moreover, thanks to its flexible observation strategy, GAME is also able to target other interesting scientific goals in the realm of General Relativity, as well as in those involving observations of selected extrasolar systems in the brown dwarf and planetary regime.
\end{abstract}

Keywords. Relativity - gravitation - astrometry - telescopes - methods: numerical - instrumentation: miscellaneous

\section{References}

Bertotti B., Iess L., \& Tortora P., Sep. 2003, Nature (London), 425, 374

Gai M., Vecchiato A., Lattanzi M. G., Ligori S., \& Loreggia D., Sep. 2009, Adv. Space Res., 44, 588

Ni W. T., 2008, International Journal of Modern Physics D, 17, 921

Turyshev S. G., Shao M., \& Nordtvedt K. Jr., May 2004, Astronomische Nachrichten, 325, 267

Vecchiato A., Lattanzi M. G., Bucciarelli B., et al., Feb. 2003, Astron. Astrophys., 399, 337

Vecchiato A., Gai M., Lattanzi M. G., Crosta M., \& Sozzetti A., Sep. 2009, Adv. Space Res., 44,579

Will C. M., 2001, Living Rev. Relativity, 2, [Online article]: cited on 14 October 2010, http://www.livingreviews.org/Articles/Volume4/2001-4will/ 